\title{
PREVALENCIA DE PARASITISMO INTESTINAL Y SUS FACTORES ASOCIADOS EN PUBLICACIONES INDEXADAS DE COLOMBIA: REVISIÓN SISTEMÁTICA 2000-2017
}

Recibido: junio del 2018

Aceptado: mayo del 2019

Diana Marcela Baena Herrera' ${ }^{1}$ Ana Marcela Fajardo Trochez ${ }^{1}$, Jorge William Flórez Amaya ${ }^{1}$, Jaiberth Antonio Cardona-Arias ${ }^{2}$

\section{Resumen}

Introducción. En Colombia, las parasitosis intestinales constituyen un problema de salud pública poco investigado (o invisibilizado), a pesar de su relevancia como trazador de pobreza. Objetivo. Analizar la prevalencia de parasitismo intestinal y sus factores asociados en publicaciones indexadas de Colombia 1990-2017. Métodos. Revisión sistemática de la literatura científica a partir de la aplicación de un protocolo ex ante de selección de artículos con 40 estrategias, según las fases de identificación, tamización, elección e inclusión de Prisma. Se garantizó la reproducibilidad de la selección de los artículos y la extracción de la información. Se evaluó la calidad metodológica con la guía Strobe. Se estimaron prevalencias generales y específicas según agentes parasitarios, etnia y edad. Resultados. Se incluyeron 20 estudios con 4.226 sujetos, principalmente de Antioquia, niños y población mestiza. La prevalencia general fue 65,9\% (IC de $95 \%=64,5-67,4$ ), con un 38,2\% (IC de $95 \%=36,3$ - 40,0) de poliparasitismo y $19,1 \%$ (IC de $95 \%=$ $16,2-21,9)$ por especies patógenas. Las infecciones más prevalentes corresponden a Blastocystis hominis con $34,0 \%$ (IC de $95 \%=32,5-35,6$ ), E. coli $27,4 \%$ (IC de $95 \%=25,8-29,0$ ), E. nana $26,2 \%$ (IC de $95 \%=24,6-27,7$ ) y E. histolytical dispar/moshkovski 15,1\% (IC de 95\% = 13,9 - 16,3); mientras que las especies de menor magnitud fueron E. hominis, C. cayatenensis, E. vermicularis y T. hominis. La infección fue 23,6\% mayor en niños indígenas en comparación con niños mestizos; en adultos no se registraron diferencias estadísticas según la etnia. Conclusión. Las investigaciones publicadas en revistas indexadas refieren una elevada prevalencia de parasitismo intestinal global, así como la alta frecuencia de algunas especies. Para el caso colombiano, las publicaciones refieren a múltiples factores asociados con la pobreza, las condiciones materiales de vida y los aspectos sociodemográficos; aunque solo se pudo metaanalizar el mayor riesgo de la población de niños e indígenas.

Palabras clave: Colombia, enfermedades parasitarias, helmintiasis, literatura de revisión como asunto, parasitosis intestinales; prevalencia.

${ }^{1}$ Estudiante de Microbiología y Bioanálisis. Universidad de Antioquia.

${ }^{2}$ MyB, MSc de Epidemiología, MSc de Economía aplicada. Candidato a Doctor en Salud Pública de la Universidad de Antioquia. Docente de la Escuela de Microbiología de la Universidad de Antioquia. 


\section{PREVALENCE OF INTESTINAL PARASITISM AND ASSOCIATED FACTORS IN INDEXED PAPERS IN COLOMBIA: SYSTEMATIC REVISION 2000-2017}

Diana Marcela Baena Herrera ${ }^{1}$, Ana Marcela Fajardo Trochez ${ }^{1}$, Jorge William Flórez Amaya ${ }^{1}$, Jaiberth Antonio Cardona-Arias ${ }^{2}$

\section{Abstract}

Introduction: In Colombia, intestinal parasitic infections are a public health problem which has little research, even with its relevance as a poverty tracer. Objective: To analyze the prevalence of intestinal parasitism and associated factors in indexed papers in Colombia 1990-2017. Method: Systematic revision of scientific literature through carrying out an ex ante protocol to select articles with 40 strategies, according to identification phases, screening, election and inclusion of prism. Reproducibility of article selection and information extraction was guaranteed. Methodological quality was evaluated with the Strobe guide. General and specific prevalences were estimated according to parasitic agents, ethnicity and age. Results: 20 studies with 4.226 subjects were included, mainly in Antioquia, in Mestizo children. The general prevalence was $65.9 \%$ (CI of $95 \%=64.5$ - 67.4), with a $38.2 \%(\mathrm{CI}$ of $95 \%=36.3$ 40.0) of polyparasitism and $19.1 \%$ (CI of $95 \%=16-2-21.9$ ) for pathogenic species. Most prevalent infections are Blastocystis hominis with a $34.0 \%$ (CI of $95 \%=32.5-35.6$ ), E. coli $27.4 \%$ (CI of $95 \%=25.8-29.0)$, E. nana $26.2 \%$ (CI of $95 \%=24.6$ - 27.7) and E. histolytica/dispar/moshkovski $15.1 \%$ (IC of $95 \%=13.9$ - 16.3); while less species where E. hominis, C. cayatenensis, E. vermicularis y $T$. hominis. The infection was $23.6 \%$ higher in indigenous children, compared to Mestizo children. Adults did not register any statistical difference in terms of ethnicity. Conclusion: Research published in indexed journals show an elevated prevalence of global intestinal parasitism, as well as the high frequency of certain species. In the case of Colombia, papers refer to multiple factors associated to poverty, material life conditions and sociodemographic aspects. It was only possible to metaanalyze the risk of children and indigenous children. 


\section{PREVALÊNCIA DA PARASITOSE INTESTINAL E SEUS FATORES ASSOCIADOS EM PUBLICAÇÕES INDEXADAS DA COLÔMBIA: REVISÃO SISTEMÁTICA 2000-2017}

Diana Marcela Baena Herrera ${ }^{1}$, Ana Marcela Fajardo Trochez ${ }^{1}$, Jorge William Flórez Amaya ${ }^{1}$, Jaiberth Antonio Cardona-Arias ${ }^{2}$

\section{Resumo}

Introdução: na Colômbia, as parasitoses intestinais constituem um problema de saúde pública pouco pesquisado ou invisibilizado, embora sua relevância como definidor de pobreza. Objetivo: analisar a prevalência de parasitose intestinal e seus fatores associados em publicações indexadas da Colômbia 1990-2017. Métodos: revisão sistemática da literatura científica a partir da aplicação de um protocolo ex ante de seleção de artigos com 40 estratégias, segundo as fases de identificação, rastreio, escolha e inclusão de Prisma. Foi garantida a reprodutibilidade da seleção dos artigos e a extração da informação. Foi evidenciada a qualidade metodológica com o guia Strobe. Foram estimadas prevalências gerais e específicas segundo agentes parasitários, etnia e idade. Resultados: foram incluídos 20 estudos com 4.226 sujeitos, principalmente de Antioquia, Colômbia, crianças e população mestiça. A prevalência geral foi $65,9 \%$ (IC de $95 \%=64,5-67,4$ ), com $38,2 \%$ (IC de $95 \%=36,3$ - 40,0) de poliparasitose e 19,1\% (IC de $95 \%=16,2$ - 21,9) por espécies patógenas. As infecções mais prevalentes correspondem a Blastocystis hominis com $34,0 \%$ (IC de $95 \%=32,5$ - 35,6), E. coli 27,4 \% (IC de $95 \%=25,8$ - 29,0), E. nana $26,2 \%$ (IC de $95 \%=24,6-27,7$ ) e E. histolytica/dispar/moshkovski $15,1 \%$ (IC de $95 \%=13,9$ - 16,3); enquanto as espécies de menor magnitude foram E. hominis, C. cayatenensis, E. vermicularis e T. hominis. A infecção foi 23,6\% maior em crianças indígenas em comparação com crianças mestiças; em adultos, não foram registradas diferenças estatísticas segundo a etnia. Conclusão: as pesquisas publicadas em revistas indexadas referem elevada prevalência de parasitose intestinal global, bem como alta frequência de algumas espécies. Para o caso colombiano, as publicações apresentam múltiplos fatores associados com a pobreza, com as condições materiais de vida e com os aspectos sociodemográficos, embora somente tenha sido materializado o maior risco da população de crianças e indígenas. 


\section{Introducción}

La parasitosis intestinal es considerada un asunto de gran relevancia en salud pública que afecta a países de todos los niveles de ingresos, siendo responsable de altas tasas de morbilidad en el ámbito mundial (1). Estas enfermedades engloban las parasitosis del tubo digestivo producidas por protozoos (protozoosis) y por helmintos (helmintiasis), ya sean nematodos, trematodos o cestodos (2). Hasta ahora se han reportado aproximadamente 300 especies de helmintos y casi 70 especies de protozoos; de estas, una baja proporción causa enfermedades a nivel mundial (3).

Según estimaciones de la Organización Mundial de la Salud, la prevalencia mundial de geohelmintos es del $24 \%$ (cerca de 1.500 millones de infectados), siendo los países de bajos ingresos los que presentan las prevalencias más altas por no disponer de sistemas de saneamiento correctos (4). En publicaciones previas se han reportado Ascaris lumbricoides, Ancylostomídeos, Trichuris trichiura y Entamoeba histolytica como los principales agentes parasitarios relacionados con altas tasas de morbilidad, con más de mil millones de infectados por estos agentes (5).

Específicamente en Latinoamérica y el Caribe, la mayoría de países presentan áreas con prevalencias de helmintiasis superiores al $20 \%$ (6). En el continente, la población en edad escolar es el principal grupo de riesgo, con cerca de $46 \mathrm{mi}$ llones de niños en alto riesgo de sufrir infecciones parasitarias (6), lo que se ha atribuido a la falta de resistencia natu- ral o adquirida por su sistema inmune inmaduro, así como otras condiciones higiénicas y sanitarias, hábitos en salud y costumbres generales de las poblaciones. En adición a estos aspectos, se debe tener presente que algunas investigaciones con niños han reportado prevalencias más elevadas; así, un estudio de Chile reportó un 76,2\% de infectados, en Perú un $65 \%$, mientras que en Venezuela fue de 63,1\% (7-9).

Además de la edad existen otros factores asociados con este grupo de infecciones como las características geográficas, culturales y económicas, las condiciones climáticas (temperatura, humedad, vientos), la densidad poblacional, las condiciones de saneamiento ambiental, la mala calidad de la vivienda (baja disponibilidad de agua potable, inadecuada disposición de excretas), los hábitos higiénicos de los individuos (higiene personal e higiene de los alimentos), el tipo de parásito y sus relaciones simbióticas, el acceso a los servicios médicos, entre otros $(1,9,10)$.

En Colombia, en el año 2005 se realizó un censo nacional donde se reportó que el $27,7 \%$ de la población del país se encontraba expuesta a algunos de los factores enunciados anteriormente, lo que favorece la presencia de altas prevalencias de parasitosis intestinal. De igual forma, durante el periodo 2012-2014 se realizó la "Encuesta nacional de parasitismo intestinal en población escolar", en este estudio las zonas de mayor riesgo fueron el cinturón árido pericaribeño con un $56,8 \%$ de infectados, la Sierra Nevada de Santa Marta con 74,0\% y la Amazonía con $81,8 \%$; las zonas de 
moderado riesgo fueron el Chocó-Magdalena con $44,3 \%$ y la Orinoquía con $21,7 \%$; y las zonas de bajo riesgo incluyeron los territorios insulares oceánicos del Caribe con 10,9\%, La Guayana con $14,7 \%$ y Norandina con 7,8\%. Además, esta encuesta permitió identificar que los parásitos de mayor frecuencia en esta población son Blastocystis spp. con $60 \%$, parásitos comensales con el $46 \%$, Tricocéfalo con el 18,4\%, E. histolytica/dispar/ moshkovski con 17\%, Giardia lamblia $15,4 \%$, A. lumbricoides $11,3 \%$ y uncinarias $6,4 \%(11)$.

Pese a los antecedentes descritos, el estudio del parasitismo intestinal en Colombia resulta exiguo frente a la magnitud y diversidad de factores asociados de esta problemática; son pocos los estudios que abarcan la población general y pese a la disponibilidad de múltiples investigaciones desarrolladas en diferentes ámbitos académicos, a la fecha no se dispone de una sistematización de las publicaciones en revistas indexadas, las cuales revisten de alta importancia en la divulgación científica al propiciar una evaluación por pares, presentar alta calidad (por los autores, los revisores y el manuscrito per se), optimizar la difusión y el intercambio del conocimiento y constituir uno de los medios más efectivos y rigurosos para la socialización de nuevos productos de conocimiento científico $(12,13)$. Esto toma mayor relevancia al considerar que el parasitismo intestinal en Colombia es un problema olvidado o invisibilizado, con pocos recursos económicos destinados para su investigación e intervención, probablemente por su baja letalidad.
En consecuencia, se realizó una revisión sistemática de publicaciones indexadas en esta temática en bases de datos científicas, ya que esta permite reunir, evaluar y sintetizar múltiples estudios, reducir los sesgos y minimizar el error aleatorio, identificar tendencias y divergencias en los resultados, obtener resultados con mayor validez externa y generar información más precisa, de mayor potencia estadística y con mejor grado de evidencia, en comparación con lo obtenido en cada estudio individual (14).

El objetivo de este estudio fue determinar la prevalencia de parasitismo intestinal y sus factores asociados en publicaciones indexadas de Colombia mediante una revisión sistemática de la literatura científica.

\section{Métodos}

\section{Tipo de estudio}

Revisión sistemática de la literatura.

\section{Protocolo de búsqueda y selección de artículos}

Según fases de la guía Prisma (Preferred Reporting Items for Systematic Reviews and Meta-Analyses) (15).

\section{Identificación}

Artículos de investigación originales publicados en Medline-PubMed (base de datos interdisciplinaria de la Biblioteca Nacional de Medicina de los Estados Unidos con más de 14 millones de referencias de artículos biomédicos desde 1950), ScienceDirect (colección elec- 
trónica más grande del mundo) y Scielo (interdisciplinaria y recoge publicaciones científicas de la comunidad de habla hispana y Latinoamérica). La identificación de los términos de búsqueda se hizo con dos estrategias, consultando los sinónimos de prevalencia y parásitos intestinales en el lenguaje controlado de los tesauros DeCS (Descriptores en Ciencias de la Salud) y MeSH (Medical Subject Headings) y por una cosecha de perlas o bola de nieve, combinando las etapas del método tradicional y el método exhaustivo, esto es, identificar revisiones $\mathrm{u}$ otros artículos del tema central, seleccionar los términos clave para su indexación, buscar otros artículos relevantes con los nuevos términos, seleccionar las bases de datos y repetir este proceso en cada nueva base de datos hasta no hallar nuevos términos.

Con estos procedimientos se cruzaron los términos prevalencia, frecuencia, incidencia y ocurrencia con parásito, parasitosis, amebiasis intestinal, entamebiasis intestinal, helmintiasis, giardiasis intestinal, infección por gusanos parásitos y helmintos, circunscrito a Colombia, para un total de 40 estrategias de búsqueda en cada base de datos. Esto evidenció la alta sensibilidad y exhaustividad de la búsqueda. Los artículos obtenidos fueron exportados a una fuente común en Excel para la eliminación de duplicados.

Algunas sintaxis utilizadas fueron las siguientes. En PubMed

i) ((prevalence[Title/Abstract]) AND parasite[Title/Abstract]) AND Colombia ii) ((frequency[Title/Abstract]) AND helminths[Title/Abstract]) AND Colombia

iii) ((incidence[Title/Abstract]) AND amebiasis[Title/Abstract]) AND Colombia

En ScienceDirect

i) Colombia AND Title, abstract, keywords: ocurrence Entamebiasis intestinal

ii) Colombia AND Title, abstract, keywords: Prevalence parasite

iii) Colombia AND Title, abstract, keywords: incidence Helminths

En Scielo

i) ((ti:((ab:(frequency)) AND (ab:(parasite))))) AND (Colombia)

ii) ((ti:((ab:(incidence)) AND (ab:(helminths)))) ) AND (Colombia)

iii) ((ti:((ab:(ocurrence)) AND (ab:(entamebiasis))))) AND (Colombia)

\section{Tamización}

Se tomaron como criterios de inclusión:

- Artículos con términos de búsqueda en el título, resumen o palabras claves

- Estudios en humanos

- Originales

- De prevalencia 
No se realizaron restricciones por idioma ni por año de publicación. En esta etapa se eliminaron los artículos duplicados y se corroboró que en el año de publicación de los artículos elegibles la revista estaba indexada por lo menos en Scielo.

\section{Elección}

Como criterios de exclusión se establecieron:

- Estudios con una muestra menor a 10 individuos

- Que no indiquen el método diagnóstico

- Artículos que no presenten numerador y denominador de la prevalencia

- Publicaciones que no se encuentren disponibles en texto completo pese a la solicitud a los autores.

\section{Inclusión}

En esta etapa se realizó la extracción de la información de los estudios, particularmente las variables título del estudio, año y lugar de publicación, tamaño de muestra, número de positivos, positivos por subgrupo como helminto, protozoos o especies parasitarias específicas y factores asociados.

\section{Análisis de la reproducibilidad y evaluación de la calidad}

La revisión de la literatura se basó en una estrategia que emplea un método exhaustivo para encontrar estudios a partir del diseño de criterios de inclusión y de exclusión, y los términos de la búsqueda. Esto lo hicieron de forma independiente tres investigadores para valorar la reproducibilidad de la selección de las investigaciones. De la misma forma se procedió para determinar la reproducibilidad de la extracción de las variables de los estudios incluidos, mediante el diseño de una base de datos diligenciada de manera independiente por tres investigadores.

Para evaluar la calidad de los estudios se aplicaron los criterios de la guía Strobe (Strengthening the Reporting of Observational studies in Epidemiology) (16).

\section{Análisis de la información}

En la síntesis cualitativa se describieron las variables con frecuencias absolutas y relativas; se estimó la prevalencia global de la infección en los artículos, así como prevalencias específicas según tipo y especie de parásitos, con sus intervalos de confianza del $95 \%$. Se comparó la prevalencia de infección según el grupo etario y la etnia mediante la Prueba Z (intervalos de confianza del $95 \%$ para la diferencia de proporciones), para estimar la fuerza de la asociación se estimaron razones de prevalencia con su intervalo del $95 \%$. Se realizó un análisis estratificado de la prevalencia de infección según edad y etnia, para identificar modificación del efecto por posibles efectos confusores o interacción entre estas variables. 


\section{Aspectos éticos}

Según la Resolución 8430 del Ministerio de Salud de 1993, este estudio corresponde a una investigación sin riesgo dado está basada en fuentes documentales.

\section{Resultados}

En la búsqueda inicial se identificaron 8.684 artículos, de los cuales se tamizaron 90 que contenían los términos de búsqueda en título o resumen y se sistematizaron 20 que cumplieron con el protocolo de búsqueda y selección (figura 1).
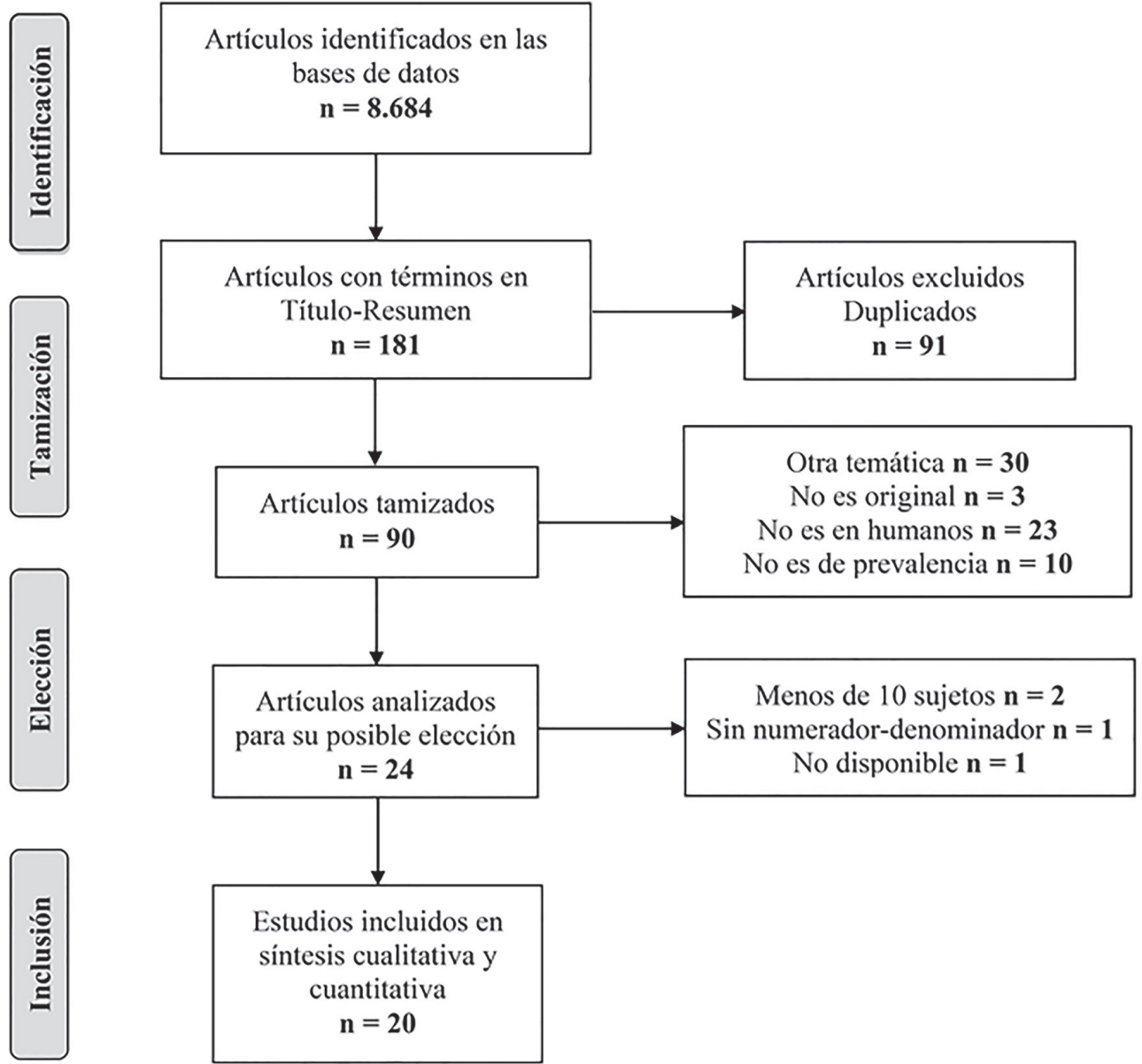

Figura 1. Flujograma de selección de los estudios Fuente: elaboración propia.

Los estudios se publicaron entre 2004 y 2013, los departamentos con mayor proporción de estudios fueron Antioquia y

104 Boyacá con un $15 \% .85 \%$ de los estudios incluyeron niños, el $15 \%$ se realizó en indígenas, las principales técnicas diagnósticas fueron el coprológico directo usado en el $85 \%$ de las publicaciones, seguido de métodos de concentración (tabla 1). 
Tabla 1. Descripción de las publicaciones según año, lugar, población y técnica

\begin{tabular}{|c|c|c|c|c|c|}
\hline Autor & Año & Departamento & Población & $\mathrm{N}$ & Técnicas \\
\hline Agudelo (17) & 2004 & Bolívar & Niños y Adultos & 382 & Directo, Ritchie \\
\hline De la Ossa (18) & 2005 & Atlántico & $\begin{array}{c}\text { Población } \\
\text { general de } \\
\text { cuatro municipios }\end{array}$ & 423 & $\begin{array}{c}\text { Directo, Ziehl- } \\
\text { Neelsen, Willis-Molloy }\end{array}$ \\
\hline Cardona (10) & 2010 & Caldas & $\begin{array}{l}\text { Adultos } \\
\text { indígenas }\end{array}$ & 132 & Directo, Ritchie \\
\hline Rodríguez (19) & 2013 & Boyacá & $\begin{array}{l}\text { Adultos, niños y } \\
\text { embarazadas }\end{array}$ & 144 & Directo, Ritchie \\
\hline Kundson (20) & 2001 & Cundinamarca & Niños & 159 & Graham \\
\hline Alvarado (21) & 2002 & Cauca & Niños & 163 & Directo, Ritchie \\
\hline Arango (22) & 2003 & Arauca & Niños & 173 & Ziehl-Neelsen \\
\hline Carmona (23) & 2004 & Antioquia & Niños & 85 & Directo, Ritchie \\
\hline Londoño24 & 2004 & Atlántico & Niños & 409 & $\begin{array}{l}\text { Directo, Éter- } \\
\text { formalina, Ziehl } \\
\text { Neelsen }\end{array}$ \\
\hline Giraldo (25) & 2005 & Quindío & Niños & 328 & Directo, Ritchie \\
\hline Londoño (26) & 2008 & Antioquia & Niños & 220 & Técnica de Ritchie \\
\hline Luna (27) & 2009 & Cundinamarca & Niños & 92 & Directo \\
\hline Arias (28) & 2010 & Quindío & Niños & 79 & Directo, Ritchie \\
\hline Manrique (29) & 2011 & Boyacá & Niños & 507 & Directo \\
\hline Suescún (30) & 2013 & Boyacá & Niños & 50 & Directo \\
\hline Garzón (31) & 2013 & Caquetá & Niños & 193 & Directo, Ritchie-Frick \\
\hline Giraldo (32) & 2013 & Risaralda & Niños & 258 & $\begin{array}{l}\text { Directo, Ritchie, } \\
\text { Graham }\end{array}$ \\
\hline Salcedo (33) & 2010 & Valle del Cauca & $\begin{array}{l}\text { Niños } \\
\text { indígenas }\end{array}$ & 57 & Directo, Sangre oculta \\
\hline Bermúdez (34) & 2012 & Valle del Cauca & $\begin{array}{l}\text { Niños } \\
\text { indígenas }\end{array}$ & 63 & $\begin{array}{c}\text { Directo, } \\
\text { Concentración, } \\
\text { Ziehl-Neelsen, } \\
\text { KatoKatz }\end{array}$ \\
\hline Cardona (35) & 2013 & Antioquia & Niños y adultos & 309 & Directo y Ritchie \\
\hline
\end{tabular}

Fuente: elaboración propia. 
La calidad metodológica fue buena en la medida en que la mayoría de estudios cumplió más de un $70 \%$ de los criterios de la guía Strobe; sin embargo, lo referido a la declaración de las limita- ciones y la discusión de las posibilidades de generalización de los resultados fue aplicado en una baja proporción de estudios (figura 2).

\begin{tabular}{|c|c|}
\hline Criterio & $\%$ de Estudio que lo cumple \\
\hline Título/ Resumen & 100 \\
\hline Fundamentación & 100 \\
\hline Objetivo & 100 \\
\hline Diseño del estudio & 100 \\
\hline Contexto & 100 \\
\hline Participantes & 95 \\
\hline Definición de variables & 100 \\
\hline Fuente de datos & 100 \\
\hline Control de sesgos & 85 \\
\hline Tamaño de muestra & 65 \\
\hline Variables cuantitativas & 100 \\
\hline Análisis estadísticos & 100 \\
\hline Resultados de los participantes & 100 \\
\hline Resultados principales & 100 \\
\hline Análisis adicionales & 80 \\
\hline Discusión de resultados clave & 100 \\
\hline Limitaciones & 20 \\
\hline Interpretaciones & 100 \\
\hline Discusión de la generalización & 25 \\
\hline Financiación & 65 \\
\hline
\end{tabular}


La población de estudio estuvo conformada por 4.226 sujetos, entre los estudios que describen sus poblaciones de estudio según el sexo, el 46,0\% ( $\mathrm{n}=$ 1.281) fueron mujeres y el 54,0\% $(\mathrm{n}=$ 1.502) hombres. La prevalencia general de parasitismo fue de $65,9 \%$ (IC de $95 \%$ $=64,5-67,4)$, con una elevada prevalencia de infección por dos o más parási- tos simultáneamente (poliparasitismo, independiente si es o no patógeno) y por especies comensales. Las infecciones más prevalentes corresponden a Blastocystis hominis, E. coli, E. nana y E. histolytica/dispar/moshkovski; mientras que las especies de menor magnitud fueron E. hominis, C. cayatenensis, E. vermicularis y T. hominis (figura 3).

\begin{tabular}{|c|c|c|c|c|c|}
\hline & $\mathbf{N}$ & Positivos & Prevalencia \% & \multicolumn{2}{|c|}{ IC $95 \%$} \\
\hline Total & 4.226 & 2.786 & 65,9 & 64,5 & 67,4 \\
\hline Poliparasitismo & 2.862 & 1.094 & 38,2 & 36,3 & 40,0 \\
\hline Monoparasitismo & 908 & 313 & 34,5 & 31,3 & 37,6 \\
\hline Comensales & 696 & 295 & 42,4 & 38,6 & 46,1 \\
\hline Patógenos & 762 & 145 & 19,1 & 16,2 & 21,9 \\
\hline Blastocystis hominis & 3.589 & 1.222 & 34,0 & 32,5 & 35,6 \\
\hline Entamoeba coli & 3.117 & 853 & 27,4 & 25,8 & 29,0 \\
\hline Endolimax nana & 3.117 & 816 & 26,2 & 24,6 & 27,7 \\
\hline Entamoeba histolitycaldispar & 3.674 & 554 & 15,1 & 13,9 & 16,3 \\
\hline Trichuris trichiura & 2.756 & 411 & 14,9 & 13,7 & 16,3 \\
\hline Giardia duodenalis & 3.774 & 558 & 14,8 & 13,6 & 15,9 \\
\hline Ascaris lumbricoides & 2.720 & 390 & 14,3 & 13,0 & 15,7 \\
\hline Cryptosporidium spp. & 1.198 & 141 & 11,8 & 9,9 & 13,6 \\
\hline Iodamoeba butschlii & 2.562 & 172 & 6,7 & 5,7 & 7,7 \\
\hline Entamoeba hartmanni & 526 & 28 & 5,3 & 3,3 & 7,3 \\
\hline Uncinarias & 1.654 & 83 & 5,0 & 3,9 & 6,1 \\
\hline Cystoisospora belli & 602 & 17 & 2,8 & 1,4 & 4,2 \\
\hline Strongyloides stercoralis & 1.039 & 28 & 2,7 & 1,7 & 3,7 \\
\hline Chilomatix mesnili & 1.636 & 38 & 2,3 & 1,6 & 3,1 \\
\hline Hymenolepis nana & 1.513 & 32 & 2,1 & 1,4 & 2,9 \\
\hline Taenia spp. & 823 & 11 & 1,3 & 0,5 & 2,2 \\
\hline Enteromonas hominis & 409 & 4 & 1,0 & 0,3 & 2,5 \\
\hline Cyclospora cayetanensis & 1.716 & 15 & 0,9 & 0,4 & 1,3 \\
\hline Enterobius vermicularis & 1.631 & 11 & 0,7 & 0,3 & 1,1 \\
\hline Trichomonas hominis & 1.114 & 7 & 0,6 & 0,1 & 1,1 \\
\hline
\end{tabular}

Figura 3. Prevalencia general, por grupo y por especie parasitaria Fuente: elaboración propia.

La prevalencia de infección fue un 14,7 \% mayor en los adultos frente a los niños; sin embargo, al estratificar los análisis según la etnia este comportamiento solo se conservó en los mestizos (prevalencia de un 16,3\% mayor en los adultos), mientras que en los indígenas se invirtió con una prevalencia de un $11,5 \%$ mayor en los niños. Por su parte, la prevalencia de infección fue 13,0 \% mayor en los indígenas; al estratificar por el grupo etario el comportamiento de la infección cambió significativamente, en la medida en que la infección resultó $23,6 \%$ mayor en niños indígenas en comparación con los niños mestizos, mientras que en los adultos no se registraron diferencias estadísticas según la etnia (tabla 2). 
Tabla 2. Análisis estratificado de la prevalencia de parasitismo intestinal según grupo etario y etnia

\begin{tabular}{|c|c|c|c|}
\hline $\begin{array}{c}\text { Variable } \\
\text { independiente }\end{array}$ & Prevalencia \% (n) & RP (IC de $95 \%)$ & $\mathrm{P}_{1}-\mathrm{P}_{2}$ (IC de $\left.95 \%\right)$ \\
\hline \multicolumn{4}{|l|}{ Grupo etario } \\
\hline Adultos $(\mathrm{N}=937)$ & $76,3(715)$ & $1,24(1,18-1,30)^{* *}$ & $14,7(11,4-18,0)^{* *}$ \\
\hline Niños (N = 2.836) & $61,6(1.747)$ & & \\
\hline Etnia & $78,2(197)$ & & \\
\hline Indígenas ( $\mathrm{N}=252$ ) & $65,1(2.589)$ & $1,20(1,08-2,48)^{* *}$ & $13,0(7,5-18,5)^{* *}$ \\
\hline Mestizos ( $\mathrm{N}=3.974$ ) & & & \\
\hline \multicolumn{4}{|l|}{ Estratificación por etnia } \\
\hline Niños indígenas $(\mathrm{N}=120)$ & $84,2(101)$ & $1,16(1,02-1,32)^{* *}$ & $11,5(0,6-22,3)^{*}$ \\
\hline Adultos indígenas $(\mathrm{N}=132)$ & $72,7(96)$ & & \\
\hline \multicolumn{4}{|l|}{ Adultos mestizos ( $\mathrm{N}=805$ ) } \\
\hline Niños mestizos ( $\mathrm{N}=2.716$ ) & $\begin{array}{c}76,9(619) \\
60,6(1.646)\end{array}$ & $1,26(1,21-1,33)^{\wedge \ldots}$ & $10,3(12,8-19,8)^{\wedge 1}$ \\
\hline \multicolumn{4}{|l|}{ Estratificación por edad } \\
\hline Indígenas niños ( $\mathrm{N}=120)$ & $84,2(101)$ & $1,39(1,28-1,51)^{* *}$ & $23,6(16,3-30,8)^{* *}$ \\
\hline Mestizos niños $(\mathrm{N}=2.716)$ & $60,6(1.646)$ & & \\
\hline \multicolumn{4}{|l|}{ Indígenas adultos ( $N=132$ ) } \\
\hline Mestizos adultos $(\mathrm{N}=805)$ & $\begin{array}{c}72,7(96) \\
76,9(619)\end{array}$ & $0,94(0,85-1,06)$ & $-4,2(-12,1 ; 4,4)$ \\
\hline
\end{tabular}

RP: Razón de Prevalencias. P1-P2: Diferencia de proporciones * $\mathrm{p}<0,05 .{ }^{* *} \mathrm{p}<0,01$.

Fuente: elaboración propia.

En relación con los factores asociados, se halló una alta heterogeneidad en la medida en que los artículos reportaron variables diferentes (infraestructura, estilo de vida, condiciones sociodemográficas, etc), o para una misma variable su definición operativa no coincidía entre estudios (por ejemplo, la mala higiene o el tipo de animales en el domicilio) o con base en análisis estadísticos disimiles (algunos con pruebas no paramétricas como la chi cuadrado y otros con medidas de asociación epidemiológica). Pese a esta limitación, se identificaron los principales factores de riesgo del parasitismo intestinal: los problemas de higiene; el mal lavado de las manos antes de comer o posterior a la deposición; la presencia de animales en la vivienda; el uso inadecuado o la ausencia de calzado; la mala calidad del agua de consumo en la vivienda; los problemas en la disposición de excreta (o ausencia de sanitario); la contaminación fecal de los alimentos o el agua de consumo; la ausencia de acueducto y alcantarillado; las malas condiciones de la vivienda; la baja escolaridad de la madre y los problemas de acceso a programas institucionales. 


\section{Discusión}

Este estudio resume y analiza la información publicada en revisas indexadas respecto a la prevalencia y a los factores asociados a la parasitosis intestinal en Colombia. Los departamentos con mayor proporción de estudios fueron Antioquia y Boyacá; para el primero se halla una convergencia con algunos índices de ciencia y tecnología del departamento, siendo el segundo en número de grupos de investigación a nivel territorial, de igual forma durante el periodo 2006-2015 se realizaron más de 800 proyectos dedicados a la innovación tecnológica y científica, donde la mayoría de sus publicaciones fueron enfocados en el área de la salud (34). Por otra parte, esta tendencia no coincide con Boyacá, pues su participación en temas de desarrollo e investigación es menor en comparación con otros departamentos, lo que evidencia un desarrollo importante de investigación en parasitismo intestinal a pesar de no disponer de altos indicadores de Ciencia e investigación (36).

En esta revisión, la prevalencia global fue de $65,9 \%$, lo que demuestra que en Colombia las cifras existentes en cuanto a parasitosis intestinal han persistido por largos periodos de tiempo. Uno de los primeros estudios descritos fue en el año 1965, donde se reportó una prevalencia del $84 \%$ en la población colombiana (37); seguidamente en el año 1980 se realizó el Estudio Nacional de Salud, donde se determinó que el $82 \%$ de la población se encontraba parasitada (38). Actualmente se manejan los datos arrojados por la Encuesta Nacional de Parasitismo Intestinal, realizada durante el pe- riodo 2012-2014, donde se encontraron frecuencias hasta del $81 \%$ en niños en edad escolar (11). Esto supone ausencia, baja cobertura o ineficiencia en los programas nacionales enfocados en el control de este tipo de infecciones y la constante exposición a factores de riesgo que promueven la persistencia de estas altas prevalencias.

La prevalencia de poliparasitismo fue del $38,2 \%$, la cual es relativamente similar a resultados de estudios realizados en otros países de Latinoamérica como Perú, que ha reportado prevalencias de poliparasitismo hasta del 58,2\% (39). Estas similitudes pueden deberse a que ambos países comparten problemas estructurales; por ejemplo, los relacionados con el acceso a agua potable u otros de la infraestructura sanitaria que, según varios estudios, está directamente asociado con la prevalencia de esta situación $(40,41)$.

El poliparasitismo facilita el progreso a infecciones crónicas que pueden agravar las manifestaciones clínicas de los individuos, especialmente en niños en edad escolar, observándose una mayor morbilidad comparada con la de la infección por un único parásito $(42,43)$. Además, estas coinfecciones dificultan el estudio y manejo clínico del paciente, por lo que se requiere mejorar todas las estrategias de control que actúan sobre los factores comunes a varias parasitosis (44).

En el mismo sentido, se encontró una alta prevalencia de parasitosis por especies comensales (42,4\%), que, aunque no representan riesgo en términos clínicos, son indicadores de la falta de inter- 
vención de las causas estructurales o los factores de riesgo de las comunidades afectadas (45).

Las infecciones más prevalentes correspondieron a Blastocystis hominis, E. coli, E. nana y E. histolytica/dispar; mientras que las especies de menor magnitud fueron E. hominis, C. cayatenensis, E. vermicularis y T. hominis. El resultado de Blastocystis hominis como la especie más prevalente coincide con los datos de la Encuesta Nacional de Parasitismo Intestinal 2012-2014 (11); aunque el papel patológico de este parásito todavía es tema de discusión, su presencia refleja las malas prácticas de higiene y la posible contaminación fecal de los alimentos (46); aunque en la actualidad se dispone de algunas evidencias sobre el rol patogénico de algunas especies (como B. hominis) y genotipos (47).

Por otra parte, algunos artículos no incluyen todas las técnicas descritas en el estudio o la tamización del parasitismo intestinal (48), lo que da paso a la publicación de datos subestimados como en el caso de E. vermicularis y Cryptosporidium spp. que presentaron bajas prevalencias, e incluso explica la ausencia de otros parásitos como lo es Dientamoeba fragilis, que no se reportó en ninguno de los artículos. Esto demuestra la importancia de incluir técnicas como el test de Graham, coloraciones como Zielh Neelsen modificada, Tricomica y Tricromica modificada, para así generar resultados más confiables (48).

En este estudio se observó una mayor 110 ocurrencia en niños indígenas (84\%), lo que concuerda con lo descrito en estudios de comunidades indígenas de países como Venezuela, donde se encontró una prevalencia de $83,5 \%$ en niños de la etnia Yukpa (49); en algunos escenarios, esto se explica por la doble condición de vulnerabilidad, bajo desarrollo inmunológico o de hábitos protectores, así como la otras propias de los territorios indígenas como no disponer de servicios sanitarios, alcantarillado, agua potable, ambientes con alta contaminación fecal y cohabitación con animales (50).

La alta prevalencia de parasitosis encontrada en este estudio es reflejo de las malas condiciones en las que viven muchas personas en todo el país; la falta de agua potable, la incorrecta eliminación de excretas, los niveles bajos de escolaridad, el tipo de vivienda, entre otras características de las comunidades más pobres (51). Mientras estas condiciones no se cambien y mejoren, esta problemática perdurará indefinidamente y es algo que se puede evitar fomentando en estas comunidades buenas prácticas higiénicas, el hervir el agua antes de consumir y el lavar correctamente los alimentos, así como unos buenos hábitos de aseo al momento de eliminar excretas y mejorar también las condiciones de infraestructura obteniendo alcantarillado, una correcta recolección de basuras, mejorar condiciones materiales de la vivienda y otras que permitan reducir la permanencia y la transmisión de estas infecciones (52).

Los niveles de desarrollo socioeconómico bajos, diferentes condiciones de vulnerabilidad sanitaria, múltiples problemas de acceso a la prestación de 
servicios de salud, entre otros aspectos concentrados en poblaciones pobres, son explicativos de las altas prevalencias de parasitismo reportadas en este estudio, así como de múltiples inequidades sociales y en salud; lo cual es común en Colombia y en otros países latinoamericanos. Esto recaba las trampas de la pobreza y un círculo vicioso en el cual la pobreza aumenta la vulnerabilidad frente a la enfermedad y, por otro lado, estas enfermedades conllevan a más pobreza al aumentar gastos de bolsillo en salud, pérdida de trabajo y otros que generan mayor inequidad y aumentan la brecha de las desigualdades sociales $(53,54)$.

Pese a la relevancia de las publicaciones indexadas en parasitismo intestinal, este estudio debe declarar como principal limitación el hecho de no sistematizar publicaciones que aparecen en bibliotecas, buscadores abiertos u otros repositorios, debido a limitaciones en su calidad, particularmente a la dificultad de descartar sesgos de selección e información. Los datos de prevalencia para algunas especies aparecen subestimadas, dado que el protocolo de búsqueda y selección no incluyó términos relacionados con agentes específicos. Además, en los análisis por grupos étnicos y etarios, no fue posible analizar otras características por problemas en la descripción de los estudios incluidos, por ejemplo el tipo de población indígena, si los mestizos compartían el territorio con indígenas (por ejemplo en estudios realizados con población rural o campesina), o subgrupos de niños (por ejemplo, de primera infancia o población escolar).
Con base en estas limitaciones, algunas recomendaciones para estudios posteriores incluyen:

- Realizar una estimación de la prevalencia de parasitismo intestinal contrastando fuentes como las encuestas nacionales, los estudios publicados en literatura gris, entre otras.

- Investigar las capacidades del país para la investigación en este campo, caracterizar grupos e investigadores con líneas de trabajo en esta temática.

- Sistematizar los estudios etiológicos o analíticos que permitan evaluar la interacción de los parásitos intestinales con la microbiota bacteriana intestinal, así como la patogénesis y clínica asociada.

Se concluye que las investigaciones publicadas en revistas indexadas refieren a una elevada prevalencia de parasitismo intestinal global, así como la alta frecuencia de algunas especies. Para el caso colombiano, las publicaciones refieren a múltiples factores asociados con la pobreza, las condiciones materiales de vida y los aspectos sociodemográficos; aunque solo se pudo metaanalizar el mayor riesgo de la población de niños e indígenas.

\section{Conflicto de intereses}

Ninguno de los autores declara conflicto de intereses para la publicación de este manuscrito. 


\section{Referencias bibliográficas}

1. Santana E. La parasitosis intestinal, un serio problema médico-social: Revisión bibliográfica. Revista Electrónica de Portales Médicos. 2009;4(21):470.

2. López M, Pérez M. Parasitosis intestinales. An Pediatr Contin. 2011;9(4):249-58.

3. Cox F. History of human parasitology. Clin Microbiol Rev [Clin Microbiol Rev. 2002;15(4):595-612.

4. Organización Mundial de la Salud. Helmintiasis transmitidas por el suelo: Datos y cifras [Internet]. 2019 [citado Noviembre 02 de 2018]. Ginebra: OMS. Disponible en: https://www.who. int/es/news-room/fact-sheets/detail/ soil-transmitted-helminth-infections

5. Lacoste E, Rosado F, Angel F, Rodríguez M, Medina I, Suárez R. Aspects on children epidemiology of intestinal parasites in Vegon Nutrias, Venezuela. Rev Cubana Hig Epidemiol. 2012;50(3):330-9.

6. Organización Panamericana de la Salud, Banco Interamericano de Desarrollo, Instituto de Vacunas Sabin. Un Ilamado a la acción: hacer frente a los helmintos transmitidos por el contacto con el suelo en Latinoamérica y el Caribe [Internet]. 2011 [citado Noviembre 02 de 2018]. Disponible en: https://www.paho.org/hq/ dmdocuments/2011/lac-report-esp-final-3-2011.pdf

7. Vidal S, Toloza L, Cansino B. Evolución de la prevalencia de enteroparasitosis en la ciudad de Talca, Región de Maule, Chile. Rev Chil Infect. 2010;27(4):336-40.

8. Jacinto $E$, Aponte $E$, Arrunátegui-Correa A. Prevalencia de parásitos intestinales en niños de diferentes niveles de educación del distrito de San Marcos, Ancash, Perú. Rev Med Hered.
9. Nastasi J. Prevalencia de parasitosis intestinales en unidades educativas de ciudad Bolívar, Venezuela. Rev Cuidarte. 2015;6(2):1077-84.

10. Cardona J, Rivera Y, Llanes O. Parasitosis intestinal y anemia en indígenas del resguardo Cañamomo-Lomaprieta, Colombia. Avenferm. 2014;32(2):235-44.

11. Ministerio de Salud y Protección Social, Universidad de Antioquia. Encuesta nacional de parasitismo intestinal en población escolar 2012-2014. Medellín: Facultad Nacional Salud Pública, Universidad de Antioquia; 2015.

12. García L, Cantillo V. La importancia de las publicaciones. Ingeniería y Desarrollo. 2015;33(1):7-8.

13. Ganga F, Paredes L, Pedraja L. Importancia de las publicaciones académicas: algunos problemas y recomendaciones a tener en cuenta. Idesia (Arica) 2015;33(4):111-9.

14. Cardona J, Higuita L, Ríos L. Revisiones sistemáticas de la literatura científica. Colombia: Ediciones Universidad Cooperativa de Colombia; 2016.

15. Moher D, Liberati A, Tetzlaff J, Altman D. Preferred reporting items for systematic reviews and meta-analyses: The PRISMA Statement. PLoS Med. 2009;6(7):e1000097. doi: 10.1371/journal.pmed.1000097.

16. Vandenbroucke J, Von Elm E, Altman D, Gøtzsche P, Mulrow C, Pocock S, et al. Strengthening the reporting of observational studies in epidemiology (STROBE): explanation and elaboration. PLoS Medicine. 2007;4:e297. Doi: 10.1371/journal. pmed.0040297

17. Agudelo S, Gómez L, Coronado S, Orozco A, Valencia C. Prevalencia de parasitosis intestinales y factores asociados en un corregimiento de la costa 
atlántica colombiana. Rev Salud Pública. 2008;10(4):633-42.

18. De la Ossa N, Falconar $A$, Llinás $H$, Romero C. Manifestaciones clínicas y factores de riesgo asociados a la infección por Cryptosporidium en pacientes de Barranquilla y tres municipios del Atlántico (Colombia). Rev Salud Uninorte Barranquilla. 2007;23(1):19-31.

19. Rodríguez A, Camacho J, Baracaldo C. Estado nutricional, parasitismo intestinal y sus factores de riesgo en una población vulnerable del municipio de Iza (Boyacá), Colombia año 2013. Rev Chil Nutr. 2016;43(1):45-53.

20. Knudson A, Lemos E, Ariza Y, Salazar $M$, Chávez M. Frecuencia de E vermicularis en una población escolar rural de Quipile, Colombia, 2001. Rev Salud Pública. 2003;5(1):87-99.

21. Alvarado B, Vásquez L. Determinantes sociales, prácticas de alimentación y consecuencias nutricionales del parasitismo intestinal en niños de 7 a 18 meses de edad en Guapi, Cauca. Biomédica. 2006;26(1):82-94.

22. Arango M, Rodríguez A, Prada N. Frecuencia de Cryptosporidium spp. en materia fecal de niños entre un mes y trece años en un hospital local colombiano. Colombia Médica. 2006;37(2):121-5.

23. Carmona J, Uscátegui $\mathrm{R}$, Correa $\mathrm{A}$. Parasitosis intestinal en niños de zonas palúdicas de Antioquia (Colombia). IATREIA. 2009;22(1):27-46.

24. Londoño J, Hernández A, Vargas C. Intestinal parasitism in day care homes in two municipalities of Atlántico department, northern Colombia. Boletín de Malariología y Salud Ambiental. 2010;50(2):251-60.

25. Giraldo J, Lora F, Henao L, Mejía S, Gómez J. Prevalence of giardiasis and intestinal parasites in pre-school children from homes being attended as part of a state programme in Armenia, Colombia. Rev Salud Pública. 2005;7(3):327-38.
26. Londoño A, Mejía S, Gómez J, Torres E, Gómez J. Prevalencia y factores de riesgo asociados a parasitismo intestinal en preescolares de zona urbana en Calarcá, Colombia. Rev Salud Pública. 2009;11(1):72-81.

27. Luna D, Camacho L, Rojas D, Bayona $M$. Frecuencia de enteroparasitosis en jardines infantiles aledaños a la cuenca baja del río Tunjuelito. Revista U.D.C.A Actualidad \& Divulgación Científica. 2010;13(1):7-15.

28. Arias J, Guzmán G, Lora F, Torres E, Gómez J. Prevalencia de protozoos intestinales en 79 niños de 2 a 5 años de edad de un hogar infantil estatal en Circasia, Quindío. Infectio. 2010;14(1):31-38.

29. Manrique F, Suescún S. Prevalencia de parasitismo intestinal y situación nutricional en escolares y adolescentes de Tunja. Rev CES Med. 2011;25(1):20-30.

30. Suescún S. Prevalencia de parásitos intestinales y factores de riesgo en escolares del colegio Chicamocha Kennedy I del municipio de Tuta, Boyacá - Colombia. Rev Univ Salud. 2013;15(2):218-24.

31. Garzón L, Álvarez L, Chicue J, López D, Mendoza C. Parasitosis intestinal y factores de riesgo en niños de los asentamientos subnormales, Florencia Caquetá, Colombia. Rev Fac Nac Salud Pública. 2015;33(2):171-80.

32. Giraldo B, Ramírez L, Henao D, Flórez M, Parra F. Estimación de la prevalencia de parásitos intestinales en niños de dos comunidades colombianas. Revista Biosalud. 2015;14(2):18-28.

33. Salcedo M, Flórez O, Bermúdez A, Hernández L, Araujo C. Prevalencia de parasitismo intestinal en menores de seis comunidades indígenas residentes en Cali, Colombia. Rev Salud Pública. 2012;14(1):156-68. 
34. Bermúdez A, Flórez O, Bolaños M, Medina J, Salcedo M. Enteroparasitismo, higiene y saneamiento ambiental en menores de seis comunidades indígenas Cali-Colombia. Rev Salud Pública. 2013;15(1):1-11.

35. Cardona J, Bedoya K. Frecuencia de parásitos intestinales y evaluación de métodos para su diagnóstico en una comunidad marginal de Medellín, Colombia. latreia. 2013;26(3):257-68.

36. Lucio, J, Guevara A, Perea G, Torralba $\mathrm{D}$, Romero I. Indicadores de ciencia y tecnología Colombia 2016. Colombia: Ántropos Ltda; 2017.

37. Carmona J, Arango E. Parásitos intestinales y "el progreso" Aproximación al tema a partir del caso del Urabá antioqueño en Colombia. Medicina Social. 2016;11(2):35-47.

38. Corredor A, Arciniegas E, Hernández C. Parasitismo intestinal. Santafé de Bogotá, D.C., Colombia: Ministerio de Salud - Instituto Nacional de Salud; 2000.

39. Flores V, Marcos L, Terashima A, Samalvides F, Gotuzzo E. Distribución de la entereoparasitosis en el altiplano peruano: Estudio en 6 comunidades rurales del departamento de Puno, Perú. Rev gastroenterol Perú. 2002;22(4).

40. Al-Delaimy A, Al-Mekhlafi $\mathrm{H}, \mathrm{Nasr}$ $\mathrm{N}$, Atroosh W, Nashiry M, Anuar T, et al. Epidemiology of intestinal polyparasitism among Orang Asli school children in rural Malaysia. PLoS neglected tropical diseases. 2014;8(8).

41. Morales N. Parasitismo intestinal y su relación con factores de riesgo en cuatro comunidades afrocolombianas. CoIombia: Pontificia Universidad Javeriana; 2010.

42. Navone G, Zonta M, Cociancic P, Garraza M, Gamboa M, Giambelluca L, et
Argentina. Rev Panam Salud Pública. 2017;41(24):1-9.

43. Pullan R, Brooker S. The health impact of polyparasitism in humans: are we under-estimating the burden of parasitic diseases? Parasitology. 2008;135(7):783-94.

44. Fernández J, Austidillo $C$, Segura L, Gómez N, Skantria A. Perfiles de poliparasitismo intestinal en una comunidad de la Amazonia colombiana. Biomédica. 2017;37(1):368-77.

45. Gomila B, Toledo R, Sanchis J. Amebas intestinales no patógenas: una visión clinicoanalítica. Enferm Infecc Microbiol Clin. 2011;29(3):20-28.

46. Cañete $R$, Rodríguez P. Infección por Blastocystis sp: revisión de la literatura. Revista Médica Electrónica, 2012;34(5):556-65.

47. Salinas J, Vildozola H. Infección por Blastocystis. Revista de Gastroenterología del Perú. 2007;27(3):264-74.

48. Magaró $H$, Uttaro A, Serra E, Ponce $P$, Echenique $C$. Técnicas de diagnóstico Parasitológico. Argentina: Universidad Nacional del Rosario; 2012.

49. Díaz I, Rivero Z, Bracho A, Castellanos M, Acurero E. Prevalencia de enteroparásitos en niños de la etnia Yukpa de Toromo, Estado Zulia, Venezuela. Rev Méd Chile. 2006;134(1):72-8.

50. Restrepo B, Restrepo M, Beltrán J, Rodríguez M, Ramírez R. Estado nutricional de niños y niñas indígenas de hasta seis años de edad en el resguardo Embera-Katío, Tierralta, Córdoba, Colombia. Biomédica. 2006;26(4):517-27.

51. Sánchez C. Una mirada a las enfermedades parasitarias en el país. Nova Unad. 2006;4(5).

52. Tabares L, González L. Prevalencia de parasitosis intestinales en niños menores de 12 años, hábitos higiénicos, caintestinales en poblaciones infantiles de 
racterísticas de las viviendas y presencia de bacterias en el agua en una vereda de Sabaneta, Antioquia, Colombia. Iatreia. 2008;21(3):253-9.

53. Martínez M. Inequidad en los sistemas de salud de América Latina: Análisis para Colombia, Argentina y México.
VISIÓN-Revista de Investigaciones UNICIENCIA. 2017;2(1):71-81.

54. Cardona J. Determinantes sociales del parasitismo intestinal, la desnutrición y la anemia: revisión sistemática. Revista Panamericana de Salud Pública. 2018;41(143):4-7. 